\title{
Detection of Dengue Virus among Children with Suspected Malaria, Accra, Ghana
}

\author{
Nicholas Amoako, Samuel Duodu, \\ Francis E. Dennis, Joseph H.K. Bonney, \\ Kwaku P. Asante, Juliana Ameh, Lydia Mosi, \\ Takaya Hayashi, Eudosia E. Agbosu, \\ Deborah Pratt, Darwin J. Operario, Barry Fields, \\ Jie Liu, Eric R. Houpt, George E. Armah, \\ Justin Stoler, Gordon A. Awandare
}

We report new molecular evidence of locally acquired dengue virus infections in Ghana. We detected dengue viral RNA among children with suspected malaria by using a multipathogen real-time PCR. Subsequent sequence analysis revealed a close relationship with dengue virus serotype 2, which was implicated in a 2016 outbreak in Burkina Faso.

$\mathrm{T}$ he accurate diagnosis of nonmalarial febrile illnesses remains a large challenge in many malaria-endemic countries (1). The etiologic agents in this context are often not identified because of nonspecific clinical symptoms and diagnostic limitations (2); for example, of 457 patients in Nigeria who were presumptively treated for malaria, only $3.9 \%$ tested positive (3). Because of the decline in malaria transmission over the past decade in many endemic areas, including Ghana, there is a critical need for a comprehensive characterization of the etiology of acute febrile illness (AFI) (4). Dengue virus infections cause symptoms that are similar to those of malaria, and considering the increasing reports of dengue outbreaks in countries neighboring Ghana (5-8), there is an increased need for dengue surveillance.

Author affiliations: University of Ghana, Legon, Ghana (N. Amoako, S. Duodu, F.E. Dennis, J.H.K. Bonney, L. Mosi, T. Hayashi, E.E. Agbosu, D. Pratt, G.E. Armah, G.A. Awandare); Kintampo Health Research Centre, Kintampo, Ghana (N. Amoako, K.P. Asante); Ledzokuku Krowor Municipal Assembly Hospital, Teshie, Accra, Ghana (J. Ameh); Tokyo Medical and Dental University, Tokyo, Japan (T. Hayashi); University of Virginia, Charlottesville, Virginia, USA (D.J. Operario, J. Liu, E.R. Houpt); Centers for Disease Control and Prevention, Atlanta, Georgia, USA (B. Fields); University of Miami, Florida, USA (J. Stoler).

DOI: https://doi.org/10.3201/eid2408.180341

\section{The Study}

To obtain a description of the pathogens causing febrile illnesses in Ghana, we conducted a hospital-based crosssectional study among children in 2 geographically distinct areas. Kintampo, in the Brong Ahafo region, is a semiurban area in the forest savanna middle belt and has a population of 42,957 . Teshie is a periurban area in the Greater Accra region that has $\approx 171,875$ residents (online Technical Appendix Figure https:/wwwnc.gov/EID/article/24/8/180341-Techapp1.pdf). We conducted the study during October 2016-July 2017, encompassing parts of the dry season (November-March) and rainy season (April -July). Children 1-15 years of age whose symptoms included fever were examined in the outpatient departments of Kintampo Municipal Hospital or Ledzokuku Krowor Municipal Assembly Hospital in Teshie. The study was approved by the ethics review committees of the Noguchi Memorial Institute for Medical Research, University of Ghana, the Ghana Health Service, and the Kintampo Health Research Centre. We recruited the patients for this study after we obtained written informed consent from their parents or guardians. The inclusion criteria were fever within the preceding 24 hours or measured axillary temperature $\geq 38^{\circ} \mathrm{C}$ occurring for $<7$ days and no severe or known chronic disease. Attending clinicians at the 2 sites screened a total of 10,234 children, and 700 were enrolled for the study on the basis of the inclusion criteria. We collected venous blood $(5 \mathrm{~mL})$ from each participant into EDTA-containing tubes (BD Vacutainer; Becton Dickinson, Franklin Lakes, NJ, USA) for malaria tests and full blood counts; the remaining blood was stored at $-80^{\circ} \mathrm{C}$ until use. All of the children were treated according to the Ghana Health Service treatment guidelines.

We randomly selected stored blood samples from 166 children diagnosed with AFI and screened the samples by using a customized multipathogen, real-time PCR-based TaqMan probe-array card (TAC; Applied Biosystems, Carlsbad, CA, USA), as described by Liu et al. (9). The AFI TAC assay simultaneously tests for 26 pathogens, including 3 protozoa, 7 bacteria, and 16 viruses (Table 1 ). Each card tests 6 samples and 2 controls (9).

As we expected, Plasmodium spp. was the predominant pathogen detected in samples from the children (36.8\% of samples tested; Table 2). Salmonella enterica 
Table 1. Pathogens tested for by using the customized AFI TaqMan array card used in study of dengue virus among 166 children with suspected malaria, Accra, Ghana, October 2016July $2017^{*}$

Pathogens

Bartonella spp.

Brucella spp.

Bundibugyo virus

Coxiella burnetii

Crimean-Congo hemorrhagic fever virus

Chikungunya virus

Dengue virus

Ebola virus

Hepatitis E virus

HIV

Lassa virus

Leishmania spp.

Leptospira spp.

Marburg virus

Nipah virus

O'nyong-nyong virus

Plasmodium spp.

Rickettsia spp.

Rift Valley fever virus

Salmonella spp.

Sudan virus

Trypanosoma brucei

West Nile virus

Yersinia pestis

Yellow fever virus

Zika virus

*Applied Biosystems, Carlsbad, CA, USA. AFI, acute febrile illness.

serovar Typhi, Rickettsia spp., Coxiella burnetii, and HIV-1 were also detected in some samples (Table 2). However, the most notable observation was the detection of dengue virus in samples from 2 children, 3 and 14 years of age, who were admitted to Ledzokuku Krowor Municipal Assembly Hospital; critical cycle threshold $\left(C_{t}\right)$ for these children was 24.40 and 19.35 , respectively (Figure 1). Furthermore, 1 of the dengue-positive samples was also positive for Plasmodium spp., providing a vivid demonstration of the complex etiology of AFI in malariaendemic areas. This observation is consistent with a recent report showing that $51 \%$ of febrile children in Ghana who were diagnosed with malaria parasitemia were coinfected with $\geq 1$ pathogen (10).

No dengue virus infections have previously been reported in Ghana, despite suspected transmission after the

Table 2. Pathogens detected in study of dengue virus among 166 children with suspected malaria by using customized AFI

TaqMan array card, Accra, Ghana, October 2016-July 2017*

Pathogen(s)

Plasmodium spp. only

Dengue virus only

Plasmodium spp. + dengue virus

Salmonella enterica serovar Typhi

Rickettsia spp.

Coxiella burnetii + Plasmodium spp.

HIV

Unidentified (negative result)

${ }^{*} \mathrm{AFI}$, acute febrile illness.

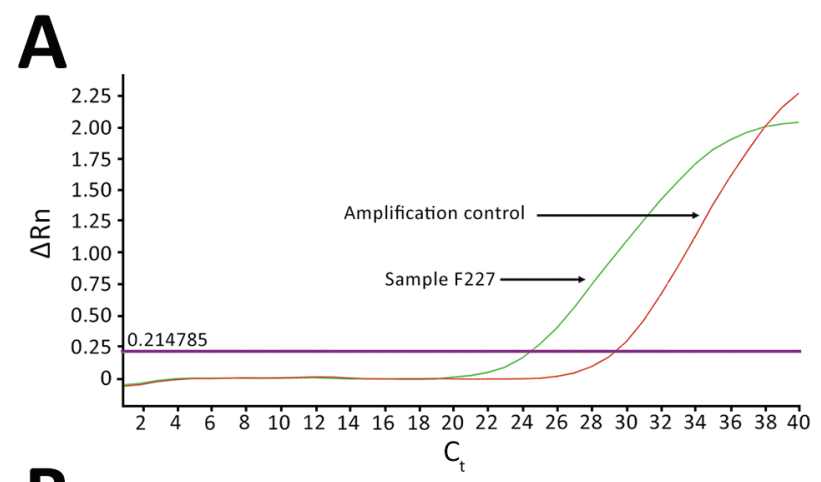

B
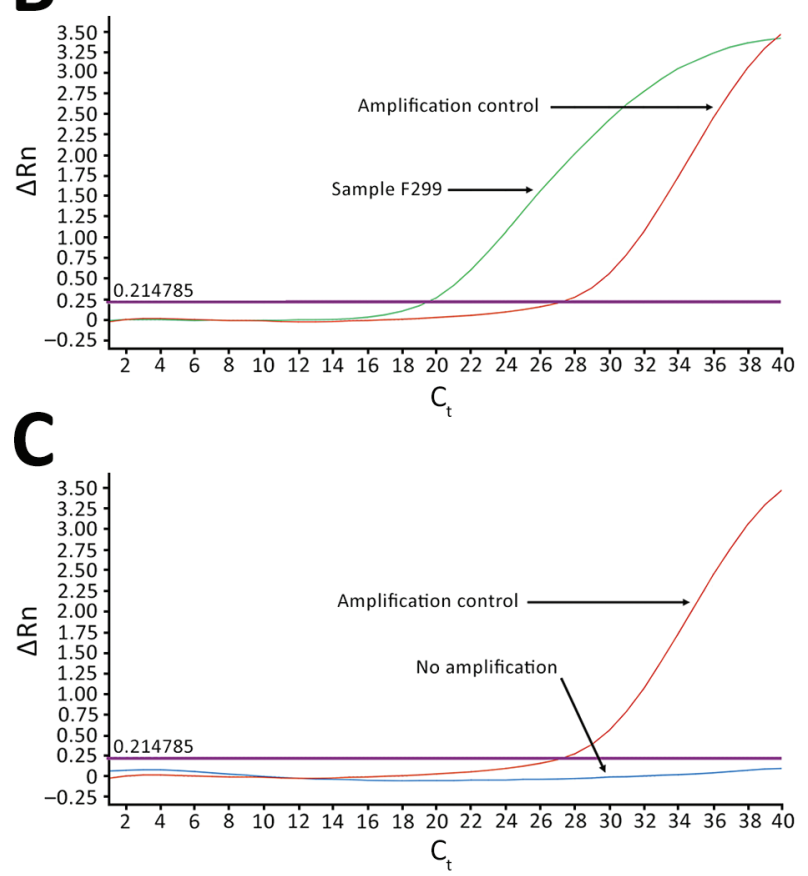

Figure 1. TaqMan array card amplification plots for 2 dengue virus-positive samples and 1 negative sample in study of dengue virus among 166 children with suspected malaria, Accra, Ghana, October 2016-July 2017. Blood samples (2.5 $\mathrm{mL}$ of the $5.0 \mathrm{~mL}$ collected) obtained from children reporting to the hospital with acute febrile illness (AFI) were screened for 26 pathogens simultaneously by using the real-time PCR TaqMan array card. The cards were in 384-well format, and each well contained $1 \mu \mathrm{L}$ of reaction mixture $(0.75 \mu \mathrm{L}$ of the extracted total nucleic acid and $0.25 \mu \mathrm{L}$ of TaqMan Fast Virus 1-step Master Mix; Life Technologies/Applied Biosystems, Foster City, CA, USA). The assays were run on an Applied Biosystems Quant Studio 7 Flex real-time PCR system, according to the manufacturer's recommendations. Two samples tested positive for dengue virus: A) F227, which amplified with critical $C_{t}$ of 24.40; and B) F299, which amplified with $C_{t}$ of 19.35 . C) All others tested negative, indicated by amplification signals $(\triangle \mathrm{Rn})$ below threshold levels at quantification cycle cutoff of 35. Each assay included nucleic acid to serve as amplification controls. External controls were spiked into each to monitor extraction and amplification efficiency, and 1 negative control was included for each batch of extraction to monitor laboratory contamination. $\mathrm{C}_{\mathrm{t}}$, cycle threshold. 
isolation of dengue virus type 2 (DENV-2) in travelers from Finland who visited Ghana during 2000-2005 (11) and our previous study showing serologic evidence of prior dengue exposure among malaria-positive children in Ghana during 2011-2014 (12). Therefore, we sought to confirm the results of the TAC screening by using 3 additional molecular methods: 1) the Trioplex TaqMan-based real-time reverse transcription PCR (RT-PCR) developed by the Centers for Disease Control and Prevention (13), which differentiates dengue from the arboviruses chikungunya (CHIKV) and Zika; 2) a second TaqMan-based real-time RT-PCR assay developed by Johnson et al. for dengue virus serotype detection (14); and 3) a conventional RT-PCR assay by using primers as described by Lanciotti et al. (15) (Figure 2). All of these methods confirmed the samples to be dengue positive. $C_{t}$ values for DENV obtained from the Trioplex assay for the 2 samples were 26.96 and 34.70; results for CHIKV and Zika virus were undetermined (negative). We recorded $\mathrm{C}_{\mathrm{t}}$ values of 27.53 and 35.42 on the Johnson et al. real-time RT-PCR assay, which also characterized the 2 samples as DENV-2.

We further sequenced viral RNA by using an ABI 3130XL Genetic Analyzer (Applied Biosystems, Carlsbad, CA, USA). BLAST search (https://blast.ncbi.nlm. nih.gov/blast.cgi) using default settings of generated nucleotide sequences revealed that the viruses in both positive samples were identical (100\% homology) and closely related to the DENV-2 strains isolated in the 2016 outbreak in Burkina Faso (GenBank accession no. KY627763.1). We deposited the nucleotide sequences of the detected virus strain in GenBank under accession nos. MG937762 and MG937763.

Apart from fever, both dengue-positive children reported chills, cough, and vomiting; however, neither reported diarrhea or rash. On a follow-up visit 2 months after enrollment, the children from whom we obtained the dengue-positive samples seemed healthy and afebrile, suggesting that no severe complications of the infection developed. Convalescent-phase blood samples from both patients tested positive for dengue-specific IgG; 1 was additionally positive for dengue IgM (Abcam human antidengue virus IgG and IgM ELISA kit; Abcam, Cambridge, UK). The parents reported that these children had not traveled outside the country, indicating that the infection was locally acquired.

\section{Conclusions}

Our previous investigations detected dengue antibodies in $21.6 \%$ of children in 3 areas of Ghana, including Kintampo and Accra; however, no virus was detected from any of them, suggesting previous exposure rather than acute infections (12). In this study, we have now confirmed the

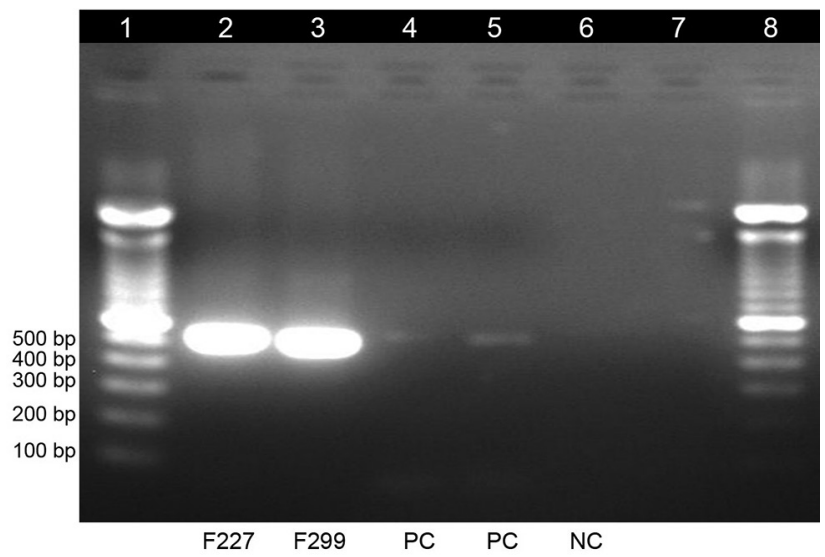

Figure 2. Gel electrophoresis of dengue virus-specific RT-PCR products in study of dengue virus among 166 children with suspected malaria, Accra, Ghana, October 2016-July 2017. We completed a conventional RT-PCR assay by using denguespecific primers from Lanciotti et al. (15) to confirm the results of the TaqMan array card assays. The amplification products (expected size $511 \mathrm{bp}$ ) were electrophoresed on $2 \%$ agarose gel, stained with ethidium bromide, and viewed under ultraviolet light. Lane 1, molecular weight marker; lanes 2 and 3, test samples; lanes 4 and 5 , positive controls; lane 6 , negative control; lane 7, empty; lane 8, molecular weight marker. RT-PCR, reverse transcription PCR.

presence of dengue virus in the blood of two children, indicating acute infections. In light of other reports from elsewhere in West Africa (5-7), this DENV-2 strain may soon become regionally endemic, if it has not already. We have advised the Ghana Health Service accordingly to take measures to intensify surveillance, consistent with prior recommendations (8).

\section{Acknowledgments}

We are grateful to the study participants for volunteering for the study and the staff of the Ledzokuku Krowor Municipal Assembly Hospital and the Kintampo Health Research Centre for their support with sample collection. We also thank Wangeci Gatei and Joel Montgomery for facilitating some aspects of the study.

This work was supported by funds from the Government of Ghana through a World Bank African Centres of Excellence grant (ACE02-WACCBIP to G.A.A.), a DELTAS Africa grant (DEL-15-007 and Wellcome Trust 107755/Z/15/Z to G.A.A.), a Royal Society Pfizer Award grant (to G.A.A.), and the Japan Agency for Medical Research and Development.

\section{About the Author}

Mr. Amoako is a PhD student at the University of Ghana, Legon. His research interests include investigating the etiology of acute febrile illness and identification of biomarkers of diseases. 


\section{References}

1. Bisoffi Z, Buonfrate D. When fever is not malaria. Lancet Glob Heal. 2013;1(1):e11-2. https://doi.org/10.1016/ S2214-109X(13)70013-5

2. Malm KL, Bart-Plange C, Armah G, Gyapong J, Adjei SO, Koram K, et al. Malaria as a cause of acute febrile illness in an urban pediatric population in Ghana. Am J Trop Med Hyg. 2012; 5(Suppl 1):401.

3. Isiguzo C, Anyanti J, Ujuju C, Nwokolo E, De La Cruz A, Schatzkin E, et al. Presumptive treatment of malaria from formal and informal drug vendors in Nigeria. PLoS One. 2014;9:e110361. http://dx.doi.org/10.1371/journal.pone.0110361

4. Stoler J, Awandare GA. Febrile illness diagnostics and the malaria-industrial complex: a socio-environmental perspective. BMC Infect Dis. 2016;16:683. http://dx.doi.org/10.1186/ s12879-016-2025-x

5. Oyero OG, Ayukekbong JA. High dengue NS1 antigenemia in febrile patients in Ibadan, Nigeria. Virus Res. 2014;191:59-61. http://dx.doi.org/10.1016/j.virusres.2014.07.023

6. Ridde V, Agier I, Bonnet E, Carabali M, Dabiré KR, Fournet F, et al. Presence of three dengue serotypes in Ouagadougou (Burkina Faso): research and public health implications. Infect Dis Poverty. 2016;5:23. http://dx.doi.org/10.1186/s40249-016-0120-2

7. Tarnagda Z, Cissé A, Bicaba BW, Diagbouga S, Sagna T, Ilboudo AK, et al. Dengue Fever in Burkina Faso, 2016. Emerg Infect Dis. 2018;24:170-2. http://dx.doi.org/10.3201/eid2401.170973

8. Stoler J, Al Dashti R, Anto F, Fobil JN, Awandare GA. Deconstructing "malaria": West Africa as the next front for dengue fever surveillance and control. Acta Trop. 2014;134:58-65. http://dx.doi.org/10.1016/j.actatropica.2014.02.017

9. Liu J, Ochieng C, Wiersma S, Ströher U, Towner JS, Whitmer S, et al. Development of a TaqMan array card for acute-febrile-illness outbreak investigation and surveillance of emerging pathogens, including Ebola virus. J Clin Microbiol. 2016;54:49-58. http://dx.doi.org/10.1128/JCM.02257-15

10. Hogan B, Eibach D, Krumkamp R, Sarpong N, Dekker D, Kreuels B, et al. Malaria coinfections in febrile pediatric inpatients : a hospital-based study from Ghana. 2018;(March):3-10. https://doi.org/10.1093/cid/cix1120

11. Huhtamo E, Uzcátegui NY, Siikamäki H, Saarinen A, Piiparinen H, Vaheri A, et al. Molecular epidemiology of dengue virus strains from Finnish travelers. Emerg Infect Dis. 2008;14:80-3. http://dx.doi.org/10.3201/eid1401.070865

12. Stoler J, Delimini RK, Bonney JHK, Oduro AR, Owusu-Agyei S, Fobil JN, et al. Evidence of recent dengue exposure among malaria parasite-positive children in three urban centers in Ghana. Am J Trop Med Hyg. 2015;92:497-500. http://dx.doi.org/10.4269/ ajtmh.14-0678

13. Centers for Disease Control and Prevention. Fact sheet for healthcare providers: interpreting Trioplex Real-time RT-PCR Assay (Trioplex rRT-PCR) results. 2017;(March). https://www.cdc.gov/ zika/pdfs/Fact-sheet-for-HCP-EUA-Trioplex-RT-PCR-Zika.pdf

14. Johnson BW, Russell BJ, Lanciotti RS. Serotype-specific detection of dengue viruses in a fourplex real-time reverse transcriptase PCR assay. J Clin Microbiol. 2005;43:4977-83. http:/dx.doi.org/ 10.1128/JCM.43.10.4977-4983.2005

15. Lanciotti RS, Calisher CH, Gubler DJ, Chang GJ, Vorndam AV. Rapid detection and typing of dengue viruses from clinical samples by using reverse transcriptase-polymerase chain reaction. J Clin Microbiol. 1992;30:545-51.

Address for correspondence: Gordon A. Awandare, University of Ghana, West African Centre for Cell Biology of Infectious Pathogens, Department of Biochemistry, Cell and Molecular Biology, PO Box LG54, Volta Road, Legon Accra, Ghana; email: gawandare@ug.edu.gh

\section{EID Podcast: Dengue Virus Transmission by Blood Stem Cell Donor after Travel to Sri Lanka; Germany, 2013}

Three days after donation of peripheral blood stem cells to a recipient with acute myeloblastic leukemia, dengue virus was detected in the donor, who had recently traveled to Sri Lanka. Transmission to the recipient, who died 9 days after transplant, was confirmed. Hematopoietic stem cell transplantation has become a major treatment option for patients with hematopoietic malignancies and immune deficiencies. Each year, approximately 50,000 allogeneic transplants are performed worldwide. Despite mandatory testing of donors and strict exclusion criteria to prevent transmission, risk remains for transmission of communicable diseases, including tropical diseases for which screening is not usually performed.
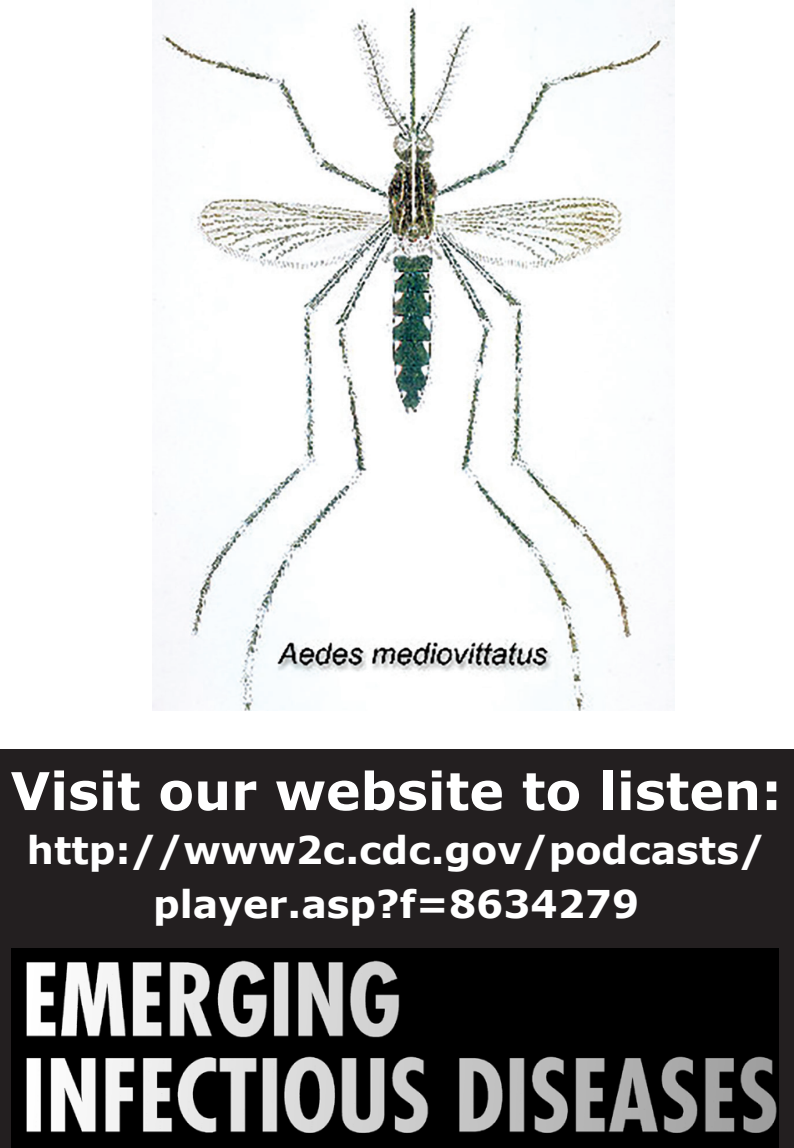